\title{
Improved Out-coupling Efficiency from a Green Microcavity OLED with a Narrow Band Emission Source
}

Tyler Fleetham, Jeremy Ecton, Guijie Li, and Jian Li*

Dr. T. Fleetham, Dr. J. Ecton, Dr. G. Li, Prof. J. Li

Department of Material Science and Engineering,

Arizona State University, Tempe, AZ 85284

E-mail: jian.li.1@asu.edu

Keywords: Microcavity, platinum complexes, light extraction, narrow spectral emission band

A highly efficient green microcavity organic light emitting diode was developed using a tetradentate cyclometalated platinum complex, PtN1N, with an intrinsically narrow emission spectral band $(\mathrm{FWHM}=18 \mathrm{~nm})$. Devices employing the narrow band emitter in MOLEDs consisting of a single pair of a high index dielectric, $\mathrm{Ta}_{2} \mathrm{O}_{5}$, and low index dielectric, $\mathrm{SiO}_{2}$, exhibited a high peak external quantum efficiency of $33.7 \%$ compared to a standard OLED in the same device architecture with a peak external quantum efficiency of $25.6 \%$. We have also found in our study that narrow band emission sources in tuned microcavity OLEDs exhibit larger enhancements in light out-coupling efficiency accompanied by small changes in color with respect to viewing angle compared to broad band emitters which is advantageous in display applications.

\section{Introduction}

Organic light emitting diodes (OLEDs) are a promising candidate for commercial applications in flat panel display technologies due to their low power consumption, low driving voltage, wide viewing angle, and high color gamut. ${ }^{[1]}$ Significant improvements in the device efficiency of OLEDs has been realized through the development of new device architectures, ${ }^{[2]}$ efficient charge transporting materials, ${ }^{[3]}$ and efficient emissive materials spanning the visible spectrum. ${ }^{[4]}$ In particular, the development of phosphorescent heavy metal complexes, which can harvest all of the electrogenerated excitons, has afforded near 
$100 \%$ electron-to-photon conversion efficiencies in select devices ${ }^{[5-6]}$ Despite such improvements, the external quantum efficiency remains limited to $20-30 \%$ for standard OLEDs on planar glass substrates since most of the generated photons do not contribute to the out-coupled optical power as a result of photon losses inside of the device. ${ }^{[7]}$ These photon losses include surface plasmon polaritions (SPPs), ${ }^{[8]}$ photon absorption at the metal electrode surface,${ }^{[9]}$ and photons trapped by total internal reflection due to the mismatch of the refractive indices between the ITO anode ( $\mathrm{n}$ 1.9) with glass ( $\mathrm{n}$ 1.5) (waveguide modes) and the mismatch of refractive indices between glass and air (n 1) (substrate modes). ${ }^{[10]}$ Thus, care must be taken to improve the light out-coupling efficiency, or fraction of photons emitted from the device to total generated photons. Strategies for this include: using high index substrates $(\mathrm{n} \geq 1.8),{ }^{[11]}$ creating surface roughness at the glass/air interface, ${ }^{[12]}$ implementing an ordered microlens array, ${ }^{[13]}$ using a periodic two-dimensional (2D) photonic crystal, ${ }^{[14]}$ or through the design of a microcavity OLED (MOLED). ${ }^{[15]}$ MOLEDs are of particular interest due to their simple fabrication and their ability to be used in conjunction with the other aforementioned strategies. ${ }^{[16]}$ A MOLED is formed by positioning the emissive layer (EML) between a highly reflective cathode and semi-reflective out-coupling mirror separated by a distance on the order of the wavelength of light emitted. Interference effects caused by the cavity redistribute the internal optical field and can change the spontaneous emission of the source inside of the cavity. ${ }^{[17]}$ With an appropriate cavity design, a preferential propagation direction of the photons can be forced from the total internal reflection regime toward the extraction cone, resulting in an increase in light out-coupling efficiency. ${ }^{[15-17]}$ Following this approach, significant improvements in efficiency as well as optical purity can be achieved with only a slight modification to the original device structure. ${ }^{[15]}$ For example, one report from Wang et al. demonstrated an EQE as high as $40 \%$ with the addition of a $\mathrm{Ta}_{2} \mathrm{O}_{5}$ capping layer and a gold anode compared to $\sim 25 \%$ using a standard OLED on a planar glass substrate with an ITO anode. ${ }^{[15 a]}$ 
It has been previously suggested that microcavity effects are stronger with narrow band emission sources since a larger fraction of the emission profile can be accelerated by the cavity. ${ }^{[18]}$ For this reason, organic emitters with narrow emission spectral bands are desired for microcavity OLEDs. However, organic emitters typically exhibit large spectral emission bands with a full width at half maximum (FWHM) between 40nm and 100nm, which makes them less suitable for a MOLED design compared with sources that exhibit a narrow spectral emission band, such as inorganic emitters or colloidal quantum dots. ${ }^{[19-21]}$ Recently, select rigid tetradentate Pt complexes were designed to exhibit extremly narrow emission bandwidths. ${ }^{[22]}$ In this report, we employ one of these narrow band emitters, PtN1N, with a FWHM of $18 \mathrm{~nm}$, into a MOLED structure. By tuning the optical cavity, ${ }^{[23]}$ a high forward viewing EQE of $33.7 \%$ was achieved compared with the reference OLED structure without a DBR which exhibited a peak EQE of $25.6 \%$, which is an enhancement factor of 1.32 . Furthermore, compared to a characteristically broad organic emitter in the same structures, PtN1N greatly outperformed the broad emitter in tuned MOLEDs, demonstrating both larger enhancement factors and smaller changes in color with respect to viewing angle. Such a development is a great advantage for many display applications.

\section{Materials and Methods}

\subsection{Materials}

The hole injection material, 1,4,5,8,9,11-hexaazatriphenylene-hexacarbonitrile (HATCN), was purchased from Lumtec Corp., the hole transporting layer, N,N'-diphyenyl-N,N'-bis(1naphthyl)-1,1'-biphenyl-4,4"-diamine (NPD), was purchased from Chemical Alta, and the materials di-[4-(N,N-di-toylyl-amino)-phyenyl]cyclohexane (TAPC), 2,6-bis(Ncarbazolyl)pyridine $(26 \mathrm{mCPy}),{ }^{[24]}$ diphenyl-bis[4-(pyridine-3-yl)phenyl]silane (DPPS), ${ }^{[25]} 1,3-$ bis(3,5-dipyrid-3-yl-phenyl)benzene (BmPyPB), ${ }^{[26]}$ platinum(II)-2'-(H-pyrazol-1-yl)-9(pyridine-2-yl)-9H-2,9'-bicarbazole (PtN1N), ${ }^{[22]}$ and platinum(II)-2-(3-(3-(pyridine-2yl)phenoxy)phenoxy)pyridine (PtOO3) ${ }^{[27]}$ were prepared following previous literature reports. 


\subsection{Device Design}

Figure 1 shows a schematic diagram of the microcavity OLED. The microcavity structure consists of a reflective aluminum cathode and a semi-reflective distributed Bragg reflector (DBR) or quarter wave stack (QWS) out-coupling mirror. The DBR consists of $\lambda / 4$ optically thick repeat pairs of a high index of refraction material, $\mathrm{Ta}_{2} \mathrm{O}_{5}(\mathrm{n}=2.2)$, and a low index of refraction material, $\mathrm{SiO}_{2}(\mathrm{n}=1.46)$. The reflectivity is tuned by varying the number of DBR pairs. ${ }^{[28]}$ According to theoretical models, for MOLEDs where the optical cavity length is equal to the peak wavelength of the source emission (i.e. a second-order microcavity), it is predicted that an out-coupling mirror reflectivity on the order of $35-45 \%$ is ideal for light outcoupling enhancement. ${ }^{[29]}$ However, the accuracy of the approximation depends on many factors such as the absorption of photons at the electrodes, self-absorption from the organic layers, the dispersion relation of the out-coupling mirror, and the spectral distribution of the source. In this study, we investigate a range out-coupling mirror reflectivities by varying the number of DBRs pairs in a second order MOLED design from one to three $\mathrm{Ta}_{2} \mathrm{O}_{5} / \mathrm{SiO}_{2}$ pairs with a reflectivity centered on the peak intrinsic emission wavelength of PtN1N. The absolute reflectance versus wavelength for the DBRs in this study were measured using a Varian Cary 5000 equipped with an integration sphere and are shown in Figure 1b. The optical cavity length was altered by varying the thickness of the hole transporting layer, NPD, in the device structure of glass/DBR(z pairs)/ITO(53nm)/HATCN(10nm)/NPD(xnm)/TAPC(10nm)/ 8\%PtN1N:26mCPy (25nm)/DPPS(10nm)/BmPyPB(45nm)/LiF(1nm)/Al(100nm), where $\mathrm{x}$ is the thickness of the NPD layer in $\mathrm{nm}$, and $\mathrm{z}$ is the number of DBR pairs $(0,1,2$, or 3$)$. The ITO thickness of 53nm was optimized to achieve a peak reflectivity centered on the peak intrinsic emission wavelength of PtN1N ( 490nm). A 100nm SiO 2 spacing layer was inserted between the ITO anode and $\mathrm{Ta}_{2} \mathrm{O}_{5}$ to extend the optical cavity closer to the intrinsic emission wavelengths without changing the electrical properties of the device. 

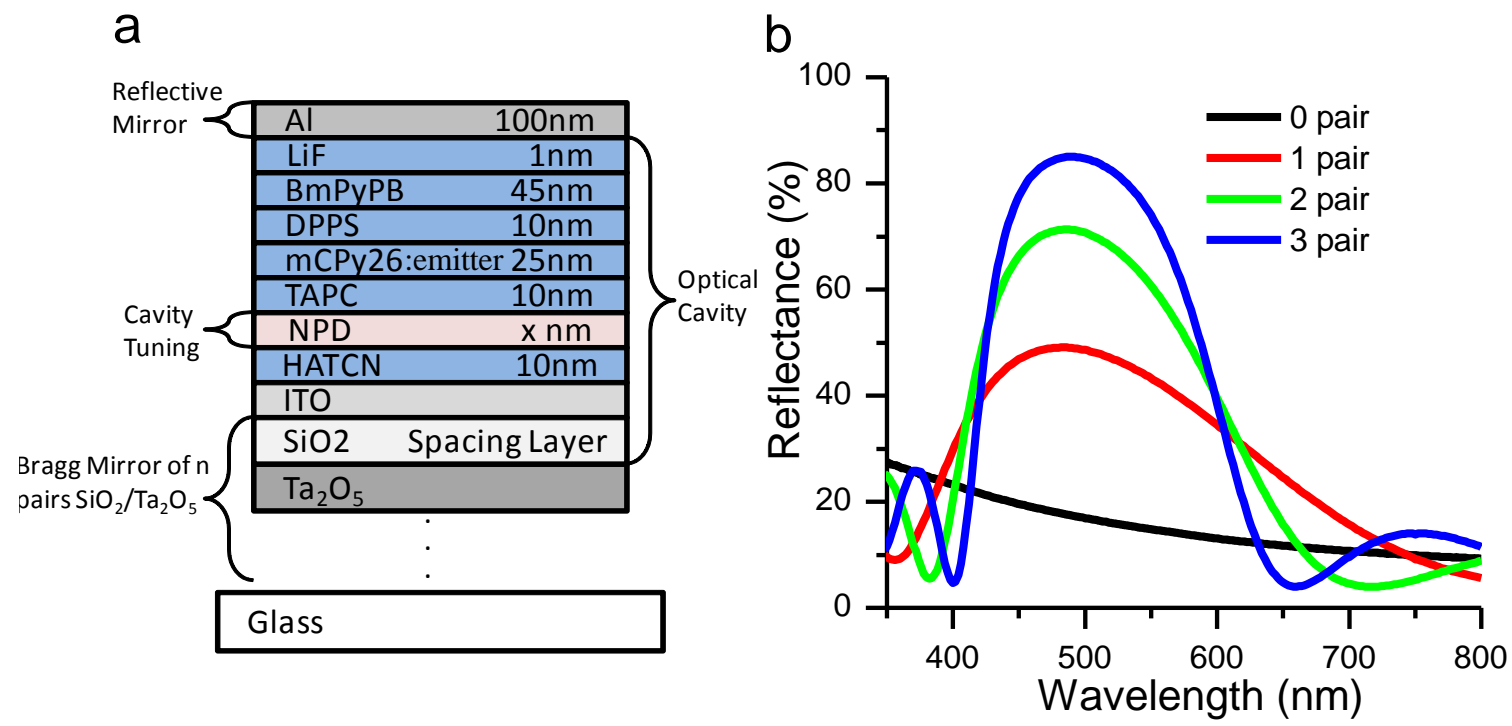

Figure 1. (a) Schematic diagram of the microcavity OLED. The optical cavity is defined between the reflective aluminum cathode mirror and the Bragg mirror, composed of alternating quarter-wavelength thick high index materials, $\mathrm{Ta}_{2} \mathrm{O}_{5}$, and low index materials, $\mathrm{SiO}_{2}$. Cavity tuning was achieved by varying the NPD layer. The emitters in this study were co-deposited with the host, $26 \mathrm{mCPy}$. (b) The reflectance versus wavelength for thin film structures of glass/ITO(60nm) (black), glass $/ \mathrm{Ta}_{2} \mathrm{O}_{5}(57 \mathrm{~nm}) / \mathrm{SiO}_{2}(100 \mathrm{~nm}) / / \mathrm{ITO}(53 \mathrm{~nm})(\mathrm{red})$, glass $/ \mathrm{Ta}_{2} \mathrm{O}_{5}(57 \mathrm{~nm}) / \mathrm{SiO}_{2}(83 \mathrm{~nm}) / \mathrm{Ta}_{2} \mathrm{O}_{5}(57 \mathrm{~nm}) / \mathrm{SiO}_{2}(100 \mathrm{~nm}) / \mathrm{ITO}(53 \mathrm{~nm})$ (green), and glass $/ \mathrm{Ta}_{2} \mathrm{O}_{5}(57 \mathrm{~nm}) / \mathrm{SiO}_{2}(83 \mathrm{~nm}) / \mathrm{Ta}_{2} \mathrm{O}_{5}(57 \mathrm{~nm}) / \mathrm{SiO}_{2}(83 \mathrm{~nm}) / \mathrm{Ta}_{2} \mathrm{O}_{5}(57 \mathrm{~nm}) / \mathrm{SiO}_{2}(100 \mathrm{~nm}) /$ ITO $(53 \mathrm{~nm})$ (blue).

\subsection{Device Fabrication and Characterization}

Thin films implementing the high-index of refraction material, $\mathrm{Ta}_{2} \mathrm{O}_{5}$, and the low-index of refraction material, $\mathrm{SiO}_{2}$, were deposited alternatingly on glass substrates in a single chamber using an ion-beam sputtering technique. The last $\mathrm{Ta}_{2} \mathrm{O}_{5}$ layer was capped with a $100 \mathrm{~nm} \mathrm{SiO}_{2}$ spacer layer. ${ }^{[30]}$ The DBR stack was then capped with a 53nm ITO layer deposited by ion assisted e-beam evaporation. The structures and naming convention that will be used in the remainder of the paper are as follows: glass $/ \mathrm{Ta}_{2} \mathrm{O}_{5}(57 \mathrm{~nm}) / \mathrm{SiO}_{2}(100 \mathrm{~nm}) / \mathrm{ITO}(53 \mathrm{~nm})(1$ pair $)$, glass $/ \mathrm{Ta}_{2} \mathrm{O}_{5}(57 \mathrm{~nm}) / \mathrm{SiO}_{2}(83 \mathrm{~nm}) / \mathrm{Ta}_{2} \mathrm{O}_{5}(57 \mathrm{~nm}) / \mathrm{SiO}_{2}(100 \mathrm{~nm}) / \mathrm{ITO}(53 \mathrm{~nm})(2 \mathrm{pair})$, glass $/ \mathrm{Ta}_{2} \mathrm{O}_{5}(57 \mathrm{~nm}) / \mathrm{SiO}_{2}(83 \mathrm{~nm}) / \mathrm{Ta}_{2} \mathrm{O}_{5}(57 \mathrm{~nm}) / \mathrm{SiO}_{2}(83 \mathrm{~nm}) / \mathrm{Ta}_{2} \mathrm{O}_{5}(57 \mathrm{~nm}) / \mathrm{SiO}_{2}(100 \mathrm{~nm}) / \mathrm{ITO}$ (53nm) (3 pair) and the reference structure of ITO on glass will be referred to as 0 pair. All substrates described were patterned using photolithography. Prior to organic depositions, the substrates were cleaned by sonication in water, acetone, and isopropanol. Organic materials were thermally evaporated at deposition rates of 0.5 to $1.5 \AA / s$ at a working pressure of less 
than $10^{-7}$ Torr. The deposition rates and thicknesses were monitored by quartz crystal microbalances. A thin $1 \mathrm{~nm} \mathrm{LiF}$ layer was deposited at rates of $<0.2 \AA / \mathrm{s}$ and aluminum cathodes were deposited at a rate of $1 \AA /$ s through a shadow mask without breaking vacuum. Individual devices had areas of $0.04 \mathrm{~cm}^{2}$.

Steady state emission experiments of the sample at room temperature as well as electroluminescent spectra of the devices were performed on a Jobin Yvon Fluorolog spectrofluorometer. I-V characteristics were taken with a Keithley 2400 source-meter and the photocurrent was measured using an OSI optoelectronics 220DP Si photodiode with a large active area of $200 \mathrm{~mm}^{2}$ to effectively collect the emitted light from the device. The responsivity of the photodiode was calibrated by the manufacturer and used without any further calibration factors. All I-V-L measurements were done in a nitrogen-filled glove-box. Normal incident emission spectra were collected at $1 \mathrm{~mA} / \mathrm{cm}^{2}$. Angular electroluminescent spectra (EL) were also measured on a Jobin Yvon Fluorolog spectrofluorometer using a rotating stage measured in ten degree increments, driven at a low constant current $(0.01$ $\mathrm{mA} / \mathrm{cm}^{2}$ to $0.001 \mathrm{~mA} / \mathrm{cm}^{2}$ ) and measured with a constant slit width. Prior to EL spectra measurements, all devices were encapsulated in a nitrogen-filled glove-box and measured in air.

\section{Results and Discussion}

The structure and photoluminescent emission spectrum of PtN1N are shown in Figure 2. PtN1N was designed with a rigid tetradentate structure and minimized vibronic features to acheive a narrow emission band with a FWHM of $18 \mathrm{~nm} .{ }^{[22]}$ 


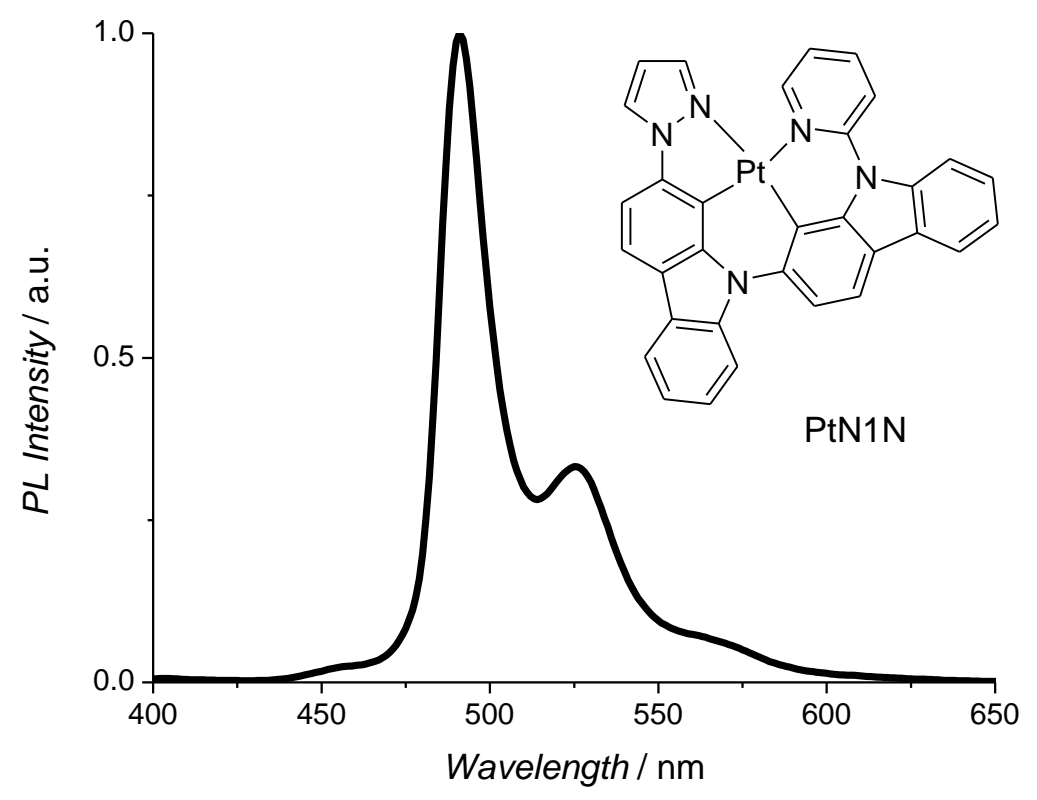

Figure 2. The emission spectrum of PtN1N in a solution of dichloromethane at room temperature, the molecular structure of which is shown in the inset.

Reference OLEDs (0 pairs) were fabricated in the structure of glass/ITO(50nm)/ HATCN(10nm)/NPD(x nm)/TAPC(10nm)/8\%PtN1N:26mCPy(25nm)/DPPS(10nm)/ $\operatorname{BmPyPB}(45 \mathrm{~nm}) / \mathrm{LiF}(1 \mathrm{~nm}) / \mathrm{Al}(100 \mathrm{~nm}) .{ }^{[31]}$ The thickness of the hole transporting layer (HTL), NPD, was varyied since increasing the thickness of the high hole mobility HTL should negligibly change the electrical characteristics, affording a correlation between the light outcoupling efficiency and the optical cavity length. Figure 3 shows the J-V curves, the EL spectra in the forward direction, and the external quantum efficiency versus current density. By implementing the effective hole and electron blockers good charge balance and a confined recombination zone are maintained. ${ }^{[31]}$ Consequently, similar $\mathrm{J}-\mathrm{V}$ characteristics are achieved with a large range of NPD thicknesses $(25 \mathrm{~nm}-60 \mathrm{~nm})$. Furthermore, the external quantum efficiency (EQE) remains approximately unchanged over most of the studied thickness range. Thus, the NPD thickness should serve well to fine tune the optical cavity length. 


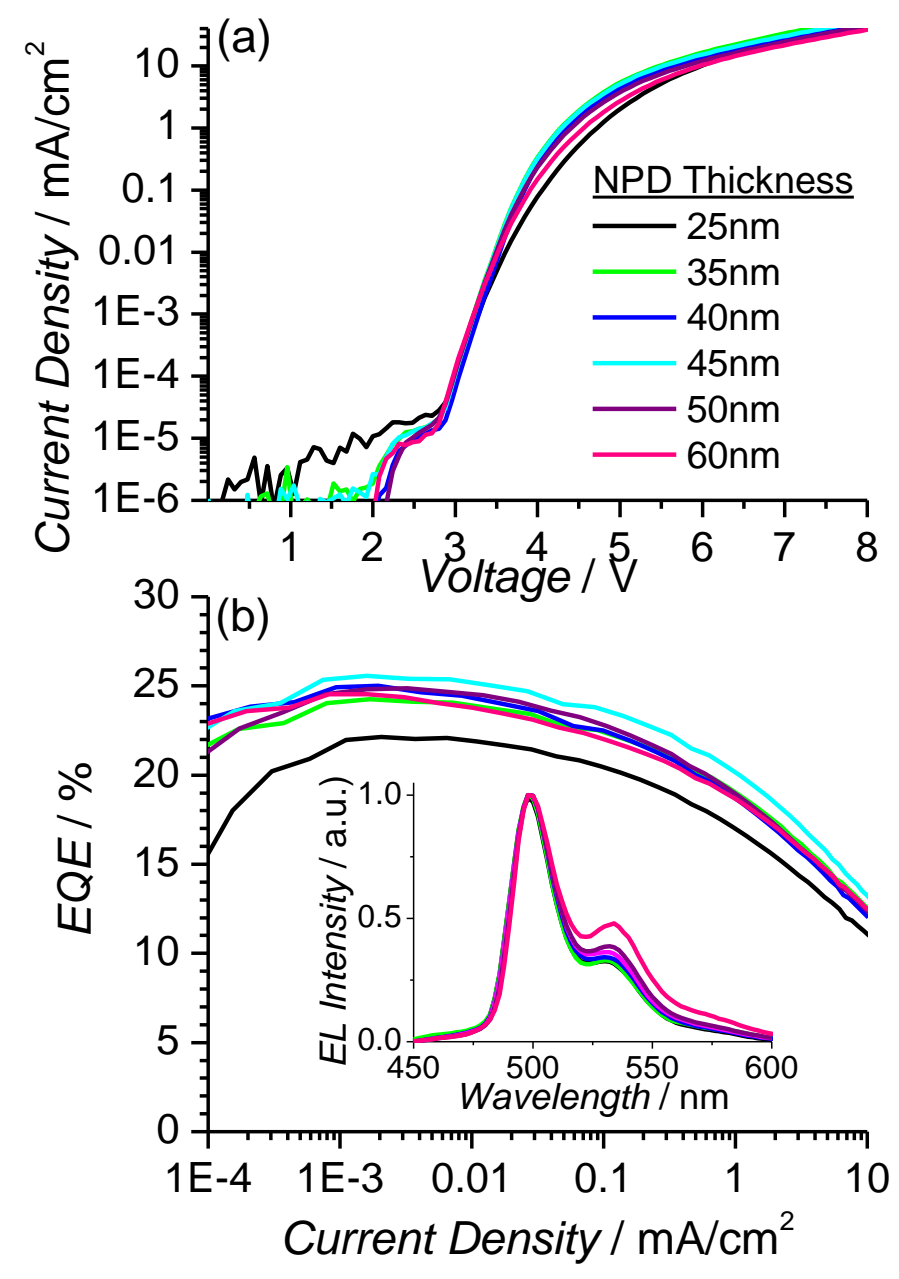

Figure 3. (a) Current density versus voltage (b) normalized electroluminescent spectra at a viewing angle of $0^{\circ}$, (c) EQE versus current density, and (d) peak EQE versus NPD thickness for PtN1N in the OLED structure of glass/ITO(50nm)/HATCN $(10 \mathrm{~nm}) / \mathrm{NPD}(\mathrm{x} \mathrm{nm}) / \mathrm{TAPC}$ (10nm)/8\%PtN1N:26mCPy $(25 \mathrm{~nm}) / \mathrm{DPPS}(10 \mathrm{~nm}) / \mathrm{BmPyPB}(45 \mathrm{~nm}) / \mathrm{LiF}(1 \mathrm{~nm}) / \mathrm{Al}(100 \mathrm{~nm})$. The emission spectra were collected at $1 \mathrm{~mA} / \mathrm{cm}^{2}$.

A series of MOLEDs were fabricated in the structure of glass/DBR(z pairs $) / \operatorname{ITO}(53 \mathrm{~nm}) /$ HATCN(10nm)/NPD(x nm)/TAPC(10nm)/8\%PtN1N:26mCPy(25nm)/DPPS(10nm)/ $\mathrm{BmPyPB}(45 \mathrm{~nm}) / \mathrm{LiF}(1 \mathrm{~nm}) / \mathrm{Al}(100 \mathrm{~nm})$, where $\mathrm{x}$ is the NPD thickness and $\mathrm{z}$ is the number of DBR pairs (0, 1, 2, or 3). Figure 4a shows the normalized electroluminescent spectra at viewing angles between $0-80^{\circ}$ for MOLEDs with a 30nm, 40nm, 50nm, and 60nm NPD layer. The corresponding CIE coordinates are in presented in Figure $\mathbf{4 b}$ and the angular intensity profiles as a function of wavelength are given in the supporting information (Figure S11Figure S13). For second-order MOLEDs, the optical cavity length is directly proportional to 
the resonant wavelength at normal incidence $\left(0^{\circ}\right)$ which is most pronounced for MOLEDs implementing 3 DBR pairs due to the high mirror reflectivity. MOLEDs consisting of 30nm NPD layer resulted in an approximately tuned microcavity structure, where the optical cavity is equal to the peak emission wavelength, with a resonance peak of $504 \mathrm{~nm}$ at normal incidence. For this tuned structure, the EL spectra at normal incidence $\left(0^{\circ}\right)$ narrows significantly from a FWHM of $24 \mathrm{~nm}$ for a single DBR pair, to $17 \mathrm{~nm}$ for $2 \mathrm{DBR}$ pairs, and $15 \mathrm{~nm}$ for 3 DBR pairs. The approximately tuned structure also only exhibited a small color shift at varying viewing angles. By increasing the NPD layer thickness a red-shift in the resonance peak was observed with a resonance peak of 522nm, 538nm, and 562nm for a 40nm, 50nm, and 60nm NPD layer, respectively. This shift is accompanied by large changes in color at different viewing angles $\left(0-80^{\circ}\right)$. 
a)

NPD Layer Thickness
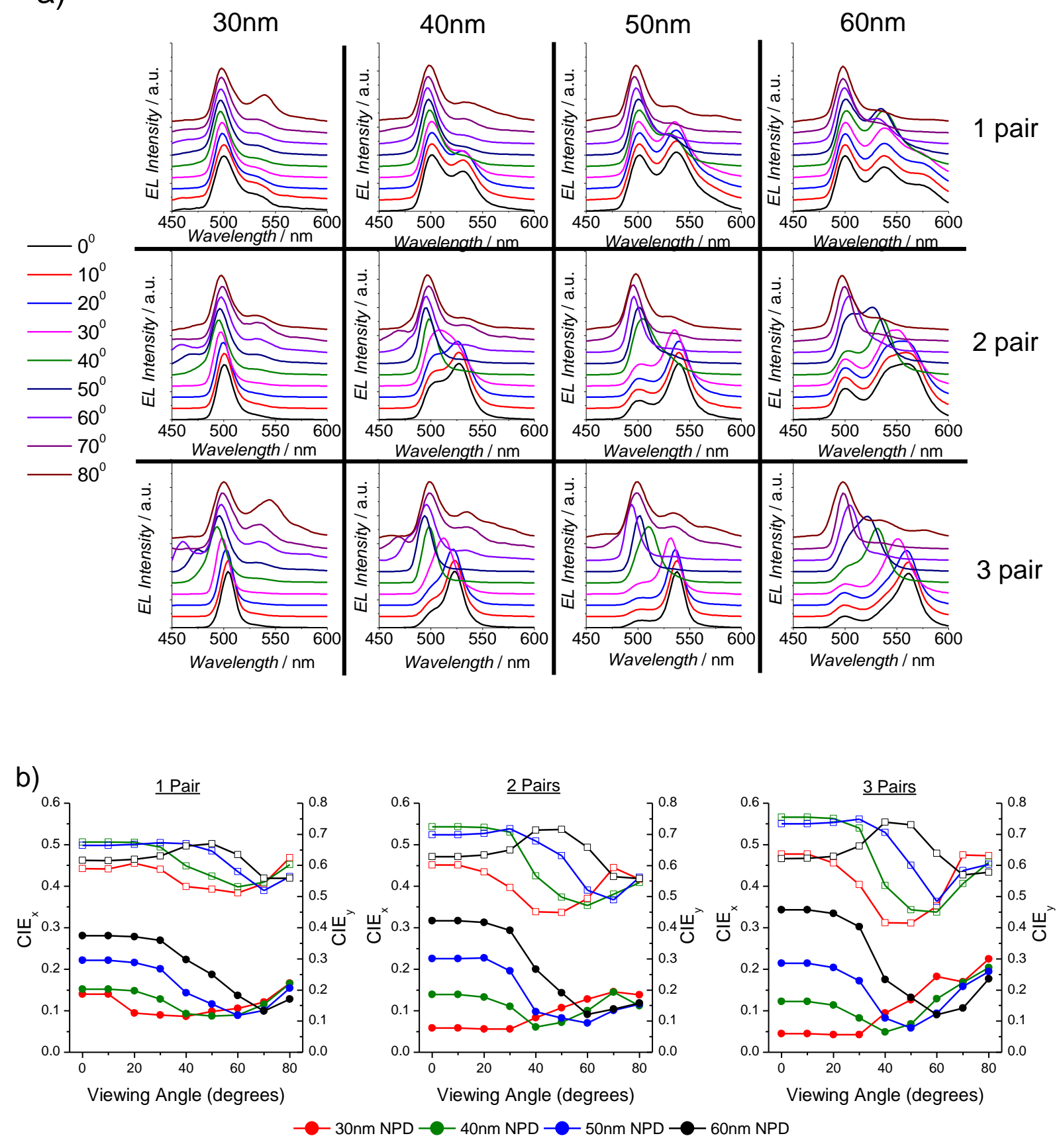

Figure 4. a) The normalized electroluminescent spectrum measured at different viewing angles between $0-80^{\circ}$ and $b$ ) the corresponding CIE coordinates for devices of the structure: glass/DBR(z pairs)/ITO/HATCN(10nm)/NPD(x nm)/TAPC(10nm)/8\%PtN1N:26mCPy $(25 \mathrm{~nm}) / \mathrm{DPPS}(10 \mathrm{~nm}) / \mathrm{BmPyPB}(45 \mathrm{~nm}) / \mathrm{LiF}(1 \mathrm{~nm}) / \mathrm{Al}(100 \mathrm{~nm})$. The devices were driven at a low constant current $\left(0.01 \mathrm{~mA} / \mathrm{cm}^{2}\right.$ to $\left.0.001 \mathrm{~mA} / \mathrm{cm}^{2}\right)$.

The external quantum efficiency versus current density for MOLEDs implementing a 40nm NPD layer thickness is shown in Figure 5(a). The series of devices were fabricated 
during the same deposition run to minimize variation in deposition conditions between each case. A peak EQE of $33.6 \%, 32.8 \%$, and $27.8 \%$ occur for MOLEDs with a single DBR pair, 2 DBR pairs, and 3 DBR pairs, respectively, compared to a peak EQE of $25 \%$ for the reference OLED without a DBR. The reduction in EQE with increasing number of DBR pairs is likely a result of increased photon absorption at the metallic cathode caused by additional round-trips inside of the optical cavity. ${ }^{[29]}$ The EQE versus NPD thickness for the MOLEDs of all 4 structures are shown in Figure 5b and Table 1. Detailed EL spectra, EQE, and power efficiency curves of the devices from which this data is drawn is provided in the Supporting Information. The peak EQE of MOLEDs with a single DBR pair exhibited an initial rise in peak efficiency with increasing NPD layer thickness to $33.7 \%$ for $45 \mathrm{~nm}$ NPD. Increasing the NPD thickness further to 50nm and 60nm resulted in a drop in EQE to $32.2 \%$ and $27.8 \%$, respectively.
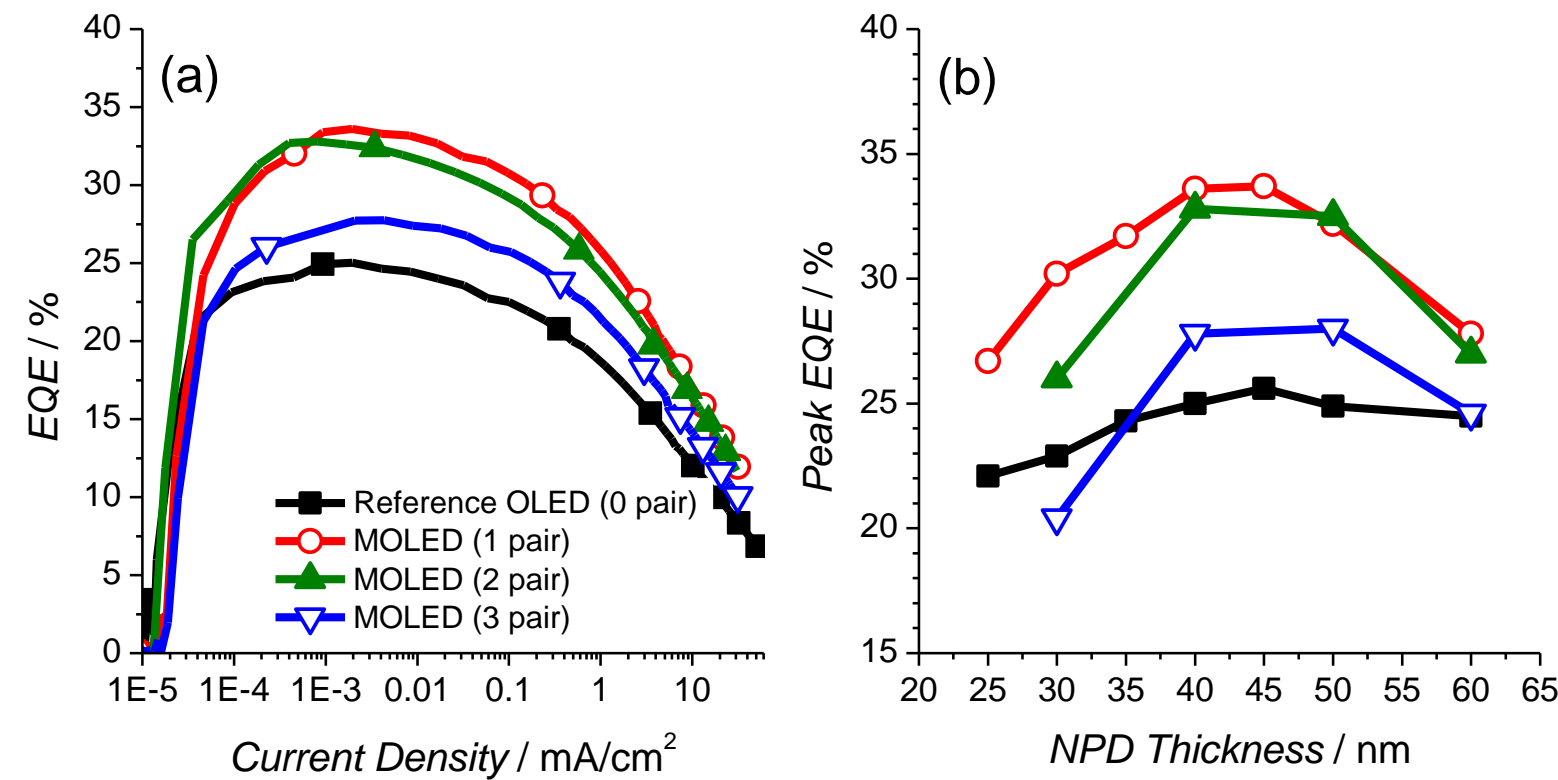

Figure 5. (a)EQE versus current density for the structure of glass/DBR(z pair)/ITO/ HATCN(10nm)/NPD(40nm)/TAPC(10nm)/8\%PtN1N:26mCPy(25nm)/DPPS(10nm)/ $\mathrm{BmPyPB}(45 \mathrm{~nm}) / \mathrm{LiF}(1 \mathrm{~nm}) / \mathrm{Al}(100 \mathrm{~nm})$ for 0 pair (squares), 1 pair (circles), 2 pair (triangles), and 3 pair (open triangles). (b) Peak EQE versus NPD thickness for devices in the above structure. 
The maximum in peak EQE with respect to NPD thickness is a result of positioning the resonant modes of the optical cavity such that the majority of emission of the source could out-couple from the cavity resonant modes. ${ }^{[32]}$ The positioning of these resonance peaks is most clearly illustrated with 3 DBR pairs in Figure 4. For the MOLED consisting of 3 DBR pairs and a 50nm NPD layer, the resonant peaks occur at 538nm, 536nm, 510nm, and 494nm for $0^{\circ}, 20^{\circ}, 40^{\circ}$, and $60^{\circ}$, respectively, which are located within the spectral distribution of the source and consequently, a high EQE is achieved. On the other hand, for the MOLEDs consisting of 3 DBR pairs and a 30nm NPD layer exhibit resonant peaks at 504nm, 502nm, $494 \mathrm{~nm}$, and $460 \mathrm{~nm}$ for $0^{\circ}, 20^{\circ}, 40^{\circ}, 60^{\circ}$, respectively, which are located at the short wavelength edge of the intrinsic spectral distribution. Similarly, for a 60nm NPD thickness, resonant peaks at $562 \mathrm{~nm}, 560 \mathrm{~nm}, 532 \mathrm{~nm}$, and $504 \mathrm{~nm}$ for $0^{\circ}, 20^{\circ}, 40^{\circ}, 60^{\circ}$, respectively, are located at the long wavelength edge of the source and contribute weakly to the overall power output coupled from the cavity. Thus, the EQE peaks at moderate NPD thicknesses, where the resonant conditions reside primarily within the range of the intrinsic emission of the source.

To explore the effect of the source spectrum, reference MOLEDs were fabricated utilizing an emitter with a spectral distribution more typical for OLED emitters (FWHM > 40nm). PtOO3, the photoluminescent spectrum of which is shown in Figure 6a, has a nearly identical emission peak wavelength to PtN1N but with a FWHM of 60nm. Thus, PtOO3 and PtN1N can be compared in identical MOLED structures. PtOO3 was implemented in the reference OLED structure of glass/ITO(50nm)/HATCN (10nm)/NPD(30 nm)/TAPC(10nm)/8\%PtOO3:26mCPy(25nm)/ DPPS(10nm)/BmPyPB(45nm)/ $\mathrm{LiF}(1 \mathrm{~nm}) / \mathrm{Al}(100 \mathrm{~nm})$ and the single DBR pair MOLED structure of glass/Ta2O5(57nm)/SiO2(100nm)/ITO(53nm)/HATCN(10nm)/NPD(30 nm)/TAPC(10nm)/ 8\%PtOO3:26mCPy(25nm)/DPPS(10nm)/BmPyPB(45nm)/LiF(1nm)/ Al(100nm). A tuned microcavity device structure with a 30nm NPD layer and a single DBR pair were chosen due to its high $\mathrm{EQE}$ and small color variation with viewing angle. The EQE versus current density 
is shown in Figure $6 \mathrm{~b}$ for the emitters PtOO3 and PtN1N in both the reference OLED and MOLED structures. The peak external quantum efficiency for devices implementing PtOO3 in the reference OLED and MOLED structure were $20.8 \%$ and $22.2 \%$, respectively. By comparison, the peak external quantum efficiency for devices implementing PtN1N in the reference OLED and MOLED structure were $22.9 \%$ and $30.2 \%$, respectively. These values correspond to an improvement in light out-coupling efficiency by 1.07 and 1.32 for MOLEDs implementing PtOO3 and PtN1N, respectively. Thus, MOLEDs implementing the narrow band emitter in the approximately tuned MOLED structure exhibited a much higher enhancement in light out-coupling efficiency than MOLEDs implementing the broad band emitter PtOO3. The smaller enhancement in light out-coupling efficiency in the case of PtOO3 was most likely attributed to the large side band emission exhibited in the thin film PL spectrum of PtOO3 which could not be accelerated by the cavity.
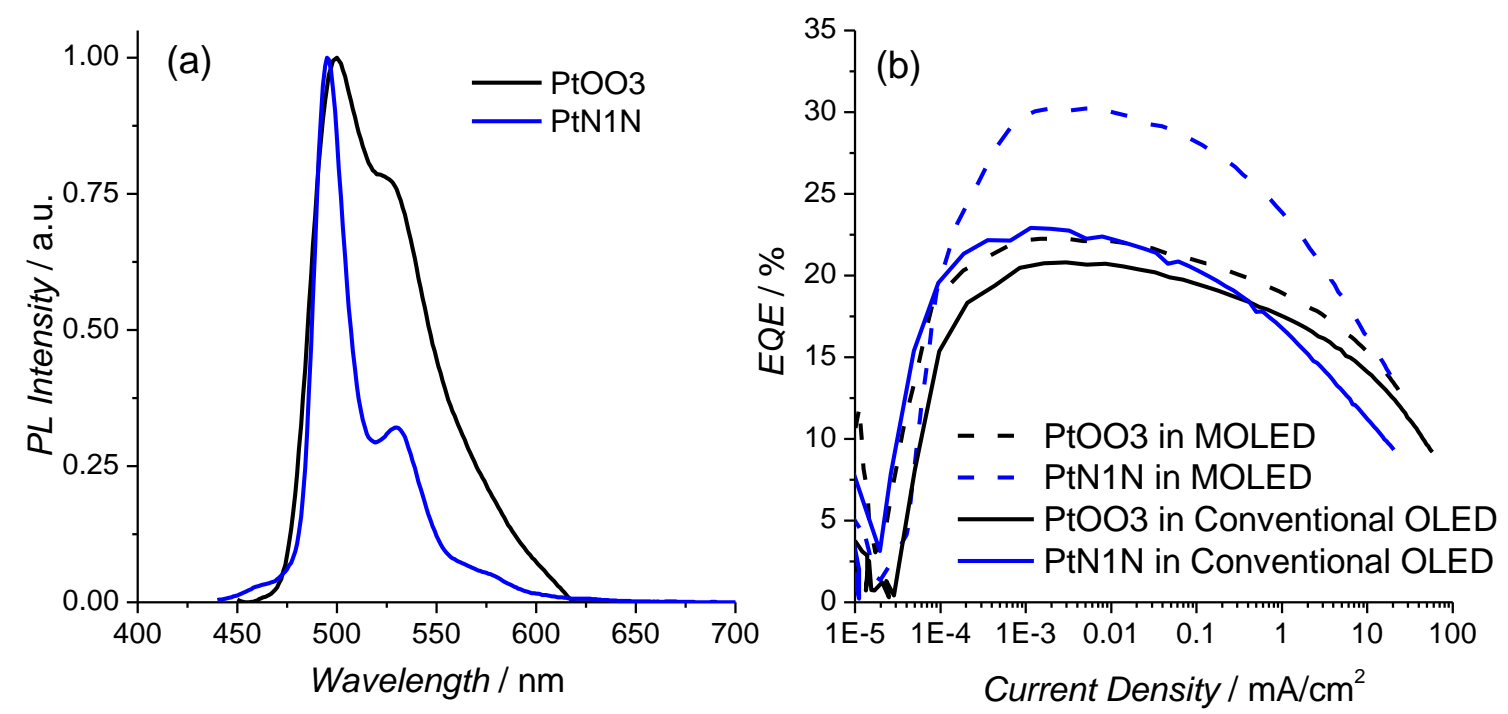

Figure 6. (a) The thin film PL spectra of PtN1N (blue) and PtOO3 (black) in a thin film of 8\%Emitter:26mCPy $(25 \mathrm{~nm}) /$ glass. (b) The external quantum efficiency of PtOO3 and PtN1N in the conventional OLED structure: glass/ITO(50nm)/HATCN(10nm)/NPD(30nm)/ TAPC (10nm)/8\%Emitter:26mCPy(25nm)/DPPS(10nm)/BmPyPB(45nm)/LiF(1nm)/ $\mathrm{Al}(100 \mathrm{~nm})$ and the 1 pair MOLED structure: glass $/ \mathrm{Ta}_{2} \mathrm{O}_{5}(57 \mathrm{~nm}) / \mathrm{SiO}_{2}(100 \mathrm{~nm}) / \mathrm{ITO}(53 \mathrm{~nm}) /$ HATCN(10nm)/NPD(30nm)/TAPC(10nm)/8\%Emitter:26mCPy(25nm)/DPPS(10nm)/ $\operatorname{BmPyPB}(45 \mathrm{~nm}) / \mathrm{LiF}(1 \mathrm{~nm}) / \mathrm{Al}(100 \mathrm{~nm})$. 
Table 1. A summary of device characteristics of PtN1N and PtOO3 in the devices fabricated from substrates with 0 DBR pair (I), 1 DBR pair (II), 2 DBR pair (III), 3 DBR pair (IV) and implementing various NPD thicknesses in the structure of glass/DBR(z-pairs)/ITO(53nm)/ HATCN(10nm)/NPD(xnm)/TAPC(10nm)/8\%Emitter:26mCPy(25nm)/DPPS(10nm)/ $\mathrm{BmPyPB}(45 \mathrm{~nm}) / \mathrm{LiF}(1 \mathrm{~nm}) / \mathrm{Al}(100 \mathrm{~nm})$, where $\mathrm{x}$ is the NPD thickness and $\mathrm{z}$ is number of pairs (0,1,2, or 3$)$.

\begin{tabular}{|c|c|c|c|c|c|c|}
\hline \multirow{2}{*}{ Structure } & \multirow{2}{*}{ Emitter } & \multirow{2}{*}{$\begin{array}{l}\text { NPD Thickness } \\
(\mathrm{nm})\end{array}$} & \multicolumn{3}{|c|}{ EQE (\%) } & \multirow{2}{*}{$\begin{array}{l}\text { Enhancement } \\
\text { (@ Peak EQE) }\end{array}$} \\
\hline & & & Peak & $100 \mathrm{~cd} / \mathrm{m}^{2}$ & $1000 \mathrm{~cd} / \mathrm{m}^{2}$ & \\
\hline 1 & PtN1N & 25 & 22.1 & 19.8 & 14.9 & NA \\
\hline 1 & PtN1N & 30 & 22.9 & 19.6 & 14.7 & NA \\
\hline 1 & PtN1N & 40 & 25 & 21.8 & 16.6 & NA \\
\hline 1 & PtN1N & 45 & 25.6 & 23.1 & 18.3 & NA \\
\hline 1 & PtN1N & 50 & 24.9 & 22.2 & 17.0 & NA \\
\hline 1 & PtN1N & 60 & 24.5 & 21.6 & 17.2 & NA \\
\hline 1 & PtOO3 & 30 & 20.8 & 19.2 & 17.0 & NA \\
\hline II & PtN1N & 25 & 26.7 & 23.1 & 17.8 & 1.21 \\
\hline II & PtN1N & 30 & 30.2 & 27.7 & $22 . .3$ & 1.32 \\
\hline II & PtN1N & 40 & 33.6 & 30.6 & 24.8 & 1.34 \\
\hline II & PtN1N & 45 & 33.7 & 31.2 & 25.9 & 1.32 \\
\hline II & PtN1N & 50 & 32.2 & 30.1 & 25.0 & 1.29 \\
\hline II & PtN1N & 60 & 27.8 & 25.5 & 21.4 & 1.13 \\
\hline II & PtOO3 & 30 & 22.2 & 20.6 & 18.2 & 1.07 \\
\hline III & PtN1N & 30 & 26.1 & 22.9 & 17.7 & 1.14 \\
\hline III & PtN1N & 40 & 32.8 & 29.8 & 24.1 & 1.31 \\
\hline III & PtN1N & 50 & 32.5 & 30.3 & 25.3 & 1.31 \\
\hline III & PtN1N & 60 & 27 & 24.9 & 21.1 & 1.1 \\
\hline IV & PtN1N & 30 & 20.4 & 17.5 & 12.9 & 0.89 \\
\hline IV & PtN1N & 40 & 27.8 & 24.7 & 19.3 & 1.11 \\
\hline IV & PtN1N & 50 & 28 & 26.3 & 21.6 & 1.12 \\
\hline IV & PtN1N & 60 & 24.6 & 23.7 & 20.1 & 1 \\
\hline
\end{tabular}

\section{Conclusion}


A systematic study of MOLEDs implementing a narrow band emission source was conducted. A high EQE was achieved with MOLEDs consisting of a single DBR pair implementing a 45nm NPD thickness with a peak EQE of $33.7 \%$ compared to the reference OLED without a DBR with a peak EQE of $25.6 \%$. Additionally, we investigated the effect of the source spectral emission band on MOLED performance by implementing two phosphorescent molecules, PtN1N (FWHM=18nm) and PtOO3 (FWHM=60nm), with similar peak emission wavelengths into identical MOLED structures. It was found that MOLEDs implementing narrow band emitters can achieve a higher light out-coupling enhancements accompanied by smaller color shifts in an approximately tuned structure compared to MOLEDs implementing broad band emitters. Our work suggests the potential for narrow band emission sources in MOLEDs and demonstrates that significant out-coupling enhancement can be achieved with a MOLED design.

\section{Acknowledgements}

T. Fleetham and J. Ecton contributed equally to this work. The authors thank the Universal Display Corporation, National Science Foundation (CHE-0748867), the Department of Energy (EE0005075), and Flexible Display Center for partial support of this work.

[1] S.R. Forrest, Nature 2004, 428, 911-918.

[2] a) N. Chopra, J. S. Swensen, E. Polikarpov, L. Cosimbescu, F. So, A. B.

Padmaperuma, Appl. Phys. Lett. 2010, 97, 033304; b) K. Walzer, B. Maennig, M. Pfeiffer, K. Leo, Chem. Rev. 2007, 107, 1233-1271; c) N. Sun, Q. Wang, Y. Zhao, Y. Chen, D. Yang, F. Zhao, J. Chen, D. Ma, Adv. Mater. 2014, 26, 1617-1621; d) Y. Zhangm J. Lee, S.R. Forrest, Nat. Commun. 2014, 5, 5008; e) N. C. Erickson, R. J. Holmes, Adv. Funct. Mater. 2013, 23, 5190-5198.

[3] a) L. Xiao, Z. Chen, B. Qu, J. Luo, S. Kong, Q. Gong, J. Kido, Adv. Mater. 2011, 23, 926-952; b) S.-H. Eom, Y. Zheng, E. Wrzesniewski, J. Lee, N. Chopra, F. So, J. Xue, Org. 
Electron. 2009, 10, 686-691; c) C. W. Lee, J. Y. Lee, Adv. Mater. 2013, 25, 596-600; d) A. Perumal, H. Faber, N. Yaacobi-Gross, P. Pattanasattayavong, C. Burgess, S. Jha, M. A. McLachlan, P. N. Stavrinou, T. D. Anthopoulos, D. D. C. Bradley, Adv. Mater. 2015, 27, 93100.

[4] a) X.C. Hang, T. Fleetham, E. Turner, J. Brooks, J. Li, Angew. Chem. Int. Ed. 2013, 52, 6753-6756; b) G. Li, T. Fleetham, J. Li, Adv. Mater. 2014, 26, 2931-2936; c) H. Uoyama, K. Goushi, K. Shizu, H. Nomura, C. Adachi, Nature 2012, 492, 234-238; d) C.-H. Yang, Y. M. Cheng, Y. Chi, C. J. Hsu, F.-C. Fang, K.-T. Wong, P.-T. Chou, C.-H. Chang, M.-H Tsai, C.-C. Wu, Angew. Chem. Int. Ed. 2007, 46, 2418-2421; e) G. Cheng, K. T. chan, W.-P. To, C.-M. Che, Adv. Mater. 2014, 26, 2540-2546; f) T. Hofback, U. Monkowius, H. Yersin, J. Am. Chem. Soc. 2015, 137, 399-404; g) Z.-Q. Zhu, T. Fleetham, E. Turner, J. Li, Adv. Mater. 2015, $27,2533-2537$.

[5] a) M. A. Baldo, D. F. O’Brien, Y. You, A. Shoustikov, S. Sibley, M.E. Thompson, S. R. Forrest, Nature 1998, 395, 151-154; b) M.A. Baldo, S. Lamansky, P.E. burrows, M.E. Thompson, S.R. Forrest, Appl. Phys. Lett. 1999, 75, 4.

[6] a) C. Adachi, M. A. Baldo, M. E. Thompson, S. R. Forrest, J. Appl. Phys. 2001, 90, 5048; b) E.L. Williams, K. Haavisto, J. Li, G.E. Jabbour, Adv. Mater. 2007, 19, 197-202; c) J. Seo, S. Lee, B. Seo, S. Moon, K. Lee, J. Park, S. Yoon, Y. Kim, Org. Electron. 2010, 11, 1759-1766; d) T. Fleetham, J. Ecton, N. Bakken, Z. Wang, J. Li, Adv. Mater. 2013, 25, $2573-$ 2576; e) T.Fleetham, L. Huang, J. Li, Adv. Funct. Mater. 2014, 24, 6066-6073.

[7] a) S. Reineke, F. Lindner, G. Schwartz, N. Seidler, K. Walzer, B. Lussem, K. Leo, Nature, 2009, 459, 234-238; b) R. Meerheim, M. Furno, S. Hofmann, B. Lussem, K. Leo, Appl. Phys. Lett. 2010, 97, 253305; d) S. Kim, J. Kim, Org. Electron. 2010, 11, 1010-1015; e) B.Krummacher, S. Nowy, J. Frischeisen, M. Klein, W. Brütting, Org. Electron. 2009, 10, 478-485. 
[8] J.-B. Kim, J.-H. Lee, C.-K. Moon, S.-Y. Kim, J.-J. Kim, Adv. Mater. 2013, 25, 3571

[9] a) C.-L. Lin, H.-W. Lin, C.-C. Wu, Appl. Phys. Lett. 2005, 87, 021101; b) W. Ji, L.

Zhang, K. Xu, W. Xie, H. Zhang, G. Liu, J. Yao, Org. Electron. 2011, 12, 2192-2197.

[10] a) S. Nowy, B.C. Krummacher, J. Frischeisen, N.A. Reinke, W. Brütting, J. Appl. Phys. 2008, 104, 123109; b) K. Hong, J.-L. Lee, Electron.Mater. Lett. 2011, 7, 77-91; c) K. Saxena, V.K. Jain, D. Mehta, Opt. Mater. 2009, 32, 221-233.

[11] a) K. Yamae, H. Tsuji, V. Kittichungchit, N. Ide, T. Komoda, J. Soc. Inf. Disp. 2014, 203; b) A. Mikami, T. Koyanagi, SID Symp. Dig. Tech. Pap. 2009, 40, 907-910; c) S.

Mladenovski, K. Neyts, D. Pavicic, A. Werner, C. Rothe, Opt. Express 2009, 17, 7562-7570.

[12] a) S. Schnitzer, E. Yablonovitch, Appl. Phys. Lett. 1993, 63, 2174-2176; b) R.

Windisch, P. Heremans, A. Knobloch, P. Kiesel, G.H. Dohler, B. Dutta, G. Borghs, Appl. Phys. Lett. 1992, 74, 2256-2262; c) T. Fujii, Y. Gao, R. Sharma, E.L. Hu, S. P. DenBaars, S. Nakamura, Appl. Phys. Lett. 2003, 84, 855-859.

[13] a) C. F. Madigan, M. H. Lu, J. C. Sturm, Appl. Phys. Lett. 2000, 76, 1650-1656; b) S. Moller, S. R. Forrest, J. App. Phys. 2002, 91, 3324; c) S.-H. Eom, E. Wrzesniewski, J. Xue, Org. Electron. 2011, 12, 472.

[14] a) Y. Do, Y. Kim, Y. Song, C. Cho, H. Jeon, Y. Lee, S. Kim, Adv. Mater. 2003, 15, 1214-1216; b) M. Fujita, T. Ueno, T. Asano, S. Noda, H. Ohhata, T. Tsuji, H. Nakada, N. Shimoji, Electron. Lett. 2003, 39, 1750-1752; c) M. Rattier, T. Krauss, J. Carlin, R. Stanley, Opt. Quant. Electron. 2002, 34, 79-89.

[15] a) Z. B. Wang, M. G. Helander, J. Qiu, D. P. Puzzo, M. T. Greiner, Z. M. Hudson, S. Wang, Z. W. Liu, Z. H. Lu, Nat. Photon. 2011, 5, 753-757; b) C. Xiang, W. Koo, F. So, H. Sasabe, J. Kido, Light: Sci. Appl. 2013, 2, e74; c) J. Lee, N. Chopra, D. Bera, S. Maslov, S.-H. Eom, Y. Zheng, P. Holloway, J. Xue, F. So, Adv. Energy Mater. 2011, 1, 174-178; d) R. Meerheim, R. Nitsche, K. Leo, Appl. Phys. Lett. 2008, 93, 043310; e) C.-L. Lin, H.-W. Lin, C.-C. Wu, Appl. Phys. Lett. 2005, 87, 021101; f) S. Dirr, S. Wiese, H. Johannes, W. 
Kowalsky, Adv. Mater. 1998, 10, 167; g) A. M. Adawi, L. G. Connolly, D. M. Whittaker, D.

G. Lidzey, E. Smith, M. Roberts, F. Qureshi, C. Foden, N. Athanassopoulou, J. Appl. Phys. 2006, 99, 54505; h) X. Y. Sun, W. L. Li, Z. R. Hong, H. Z. Wei, F. X. Zang, L. L. Chen, Z.

Shi, D. F. Bi, B. Li, Z. Q. Zhang, Z. Z. Hu, J. Appl. Phys. 2005, 97, 103112.

[16] C.-J. Yang, S.-H. Liu, H.-H. Hsieh, C.-C. Liu, T.-Y. Cho, C.-C. Wu, Appl. Phys. Lett. 2007, 91, 253508 .

[17] a) D. Delbeke, R. Bockstaele, P. Bienstman, R. Baets, H. Benisty, IEEE Sel. Top. Quantum Electron. 2002, 8, 189-206; b) E.F. Schubert, Y.H. Wang, A.Y. Chao, L.W. Tu, Appl. Phys. Lett. 1992, 60, 921-923; c) M. Suzuki, H. Yokoyama, S. D. Brorson, E. P. Ippen, Appl. Phys. Lett. 1991, 58, 998; d) J. Gruner, F. Cacialli, I, R. H. Friend, J. Appl. Phys. 1996, 80,207

[18] R. H. Jordan, L. Rothberg, A. Dodabalapur, R. E. Slusher, Appl. Phys. Lett. 1996, 69, 1997-2001.

[19] Y. Shirasaki, G. J. Supran, M. G. Bawendi, V. Bulović, Nature Photonics 2013, 7, 13

[20] a) K-H. Lee, J-H. Lee, W-S. Song, H. Ko, C. Lee, J-H. Lee, H. Yang, ACS Nano, 2013, 7, 7295; b) W. K. Bae, Y.-S. Park, J. Lim, D. Lee, L. A. Padilha, H. McDaniel, I. Robel, C. Lee, J. M. Pietryga, V. I. Klimov, Nat. Commun. 2013, 4, 2661.

[21] X. Zhong, Y. Feng, W. Knoll, M. Han, J. Am. Chem. Soc. 2003, 125, 13599-13563.

[22] a) G. Li, T. Fleetham, E. Turner, X.-C. Hang, J. Li, Adv. Optical Mater. 2015, 3, 390397; b) T. Fleetham, G. Li, L. Wen, J. Li, Adv. Mater. 2015, 26, 7116-7121.

[23] a) C. Xiang, W. Koo, F. So, H. Sasabe, J. Kido, Light: Sci. Appl. 2013, 2, e74; b) R. Bockstaele, T. Coosemans, C. Sys, L. Vanwassenhove, A. Hove, B. Dhoedt, I. Moerman, P. Van Daele, R. Baets, R. Annen, H. Melchior, J. Hall, P. Heremans, M. Brunfaut, J. Campenhout, IEEE Sel. Top. Quantum Electron. 1999, 5, 1077.

[24] E.L. Williams, K. Haavisto, J. Li, G.E. Jabbour, Adv. Mater. 2007, 19, 197-202.

[25] L. Xiao, S. Su, Y. Agata, H. Lan, J. Kido, Adv. Mater. 2009, 21, 1271-1274 
[26] H. Sasabe, E. Gonmori, T. Chiba, Y. Li, D. Tanaka, S. Su, T. Takeda, Y. Pu, K. Nakayama, J. Kido, Chem. Mater. 2008, 20, 5951-5953.

[27] E. Turner, N. Bakken, J. Li, Inorg. Chem. 2013, 52, 7344-7351.

[28] H. Kim, S. Cho, J. Oh, Y. Lee, J. Lee, J. Lee, S. Kim, Y. Park, J. Park, Y. Do, Org. Electron. 2010, 11, 137-145.

[29] a) H. Besnity, H. De Neve, C. Weisbach, IEEE Sel. Top. Quantum Electron. 1998, 34, 1612; b) J. A. E. Wasey, W. L. Barnes, J. Mod. Opt. 2000, 47, 725.

[30] A. Dodabablapur, L. J. Rothberg, R. H. Jordan, T. M. Miller, R. E. Slusher, J. M. Phillips, J. Appl. Phys. 1996, 80, 6954-6965.

[31] a) J. Lee, N. Chopra, S. Eom, Y. Zheng, J. Xue, F. So, J. Shi, Appl. Phys. Lett. 2008, 93, 123306; b) T. Fleetham, Z. Wang, J. Li, Org. Electron. 2012, 13, 1430-1435; c) T. Fleetham, G. Li, L. Wen, J. Li, Adv. Mater. 2014, 26, 7116-7121.

[32] R. E. Slusher, C. Weisbuch, Solid State Commun. 1994, 92, 149. 
Graphical Abstract

Graphical Abstract

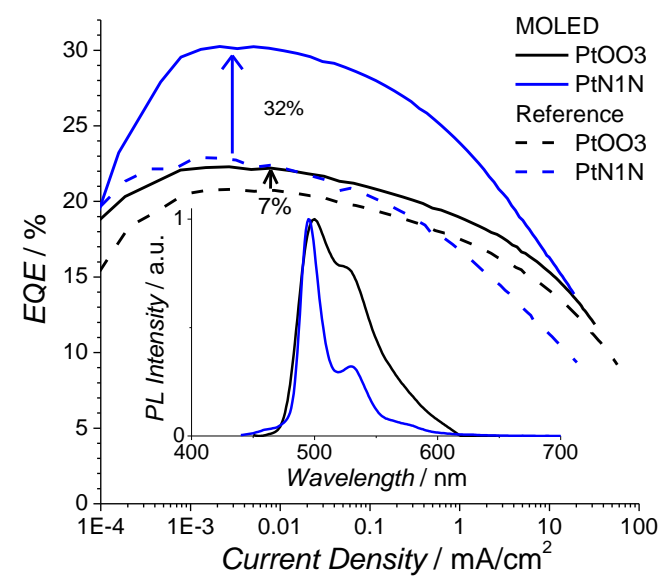

